Bull. Korean Math. Soc. 50 (2013), No. 2, pp. 687-696

http://dx.doi.org/10.4134/BKMS.2013.50.2.687

\title{
REDUCING SUBSPACES FOR TOEPLITZ OPERATORS ON THE POLYDISK
}

\author{
Yanyue Shi And Yufeng Lu
}

\begin{abstract}
In this note, we completely characterize the reducing subspaces of $T_{z_{1}^{N} z_{2}^{M}}$ on $A_{\alpha}^{2}\left(D^{2}\right)$ where $\alpha>-1$ and $N, M$ are positive integers with $N \neq M$, and show that the minimal reducing subspaces of $T_{z_{1}^{N} z_{2}^{M}}$ on the unweighted Bergman space and on the weighted Bergman space are different.
\end{abstract}

\section{Introduction}

Let $D$ denote the open unit disk in the complex plane. For $-1<\alpha<+\infty$, $L^{2}\left(D, d A_{\alpha}\right)$ is the space of functions on $D$ which are square integrable with respect to the measure $d A_{\alpha}(z)=(\alpha+1)\left(1-|z|^{2}\right)^{\alpha} d A(z)$, where $d A$ denotes the normalized Lebesgue area measure on $D . L^{2}\left(D, d A_{\alpha}\right)$ is a Hilbert space with the inner product $\langle f, g\rangle_{\alpha}=\int_{D} f(z) \overline{g(z)} d A_{\alpha}$. The weighted Bergman space $A_{\alpha}^{2}$ is the closed subspace of $L^{2}\left(D, d A_{\alpha}\right)$ consisting of analytic functions on $D$. If $\alpha=0, A_{0}^{2}$ is the Bergman space. We write $A^{2}=A_{0}^{2}$. It is known that $\left\{\frac{z^{n}}{\left\|z^{n}\right\|_{\alpha}}\right\}_{n=0}^{+\infty}$ is an orthogonal basis of $A_{\alpha}^{2}(D)$. Let $\gamma_{n}=\left\|z^{n}\right\|_{\alpha}=\sqrt{\frac{n ! \Gamma(2+\alpha)}{\Gamma(2+\alpha+n)}}$ for $n=0,1,2, \ldots$. Therefore,

$$
\|f\|_{\alpha}^{2}=\sum_{n=0}^{+\infty} \gamma_{n}^{2}\left|a_{n}\right|^{2}<\infty
$$

with $f(z)=\sum_{n=0}^{+\infty} a_{n} z^{n} \in A_{\alpha}^{2}(D)$.

Denote the unit polydisk by $D^{n}$. The weighted Bergman space $A_{\alpha}^{2}\left(D^{n}\right)$ is then the space of all holomorphic functions on $L^{2}\left(D^{n}, d v_{\alpha}\right)$, where $d v_{\alpha}(z)=$ $d A_{\alpha}\left(z_{1}\right) \cdots d A_{\alpha}\left(z_{n}\right)$. For multi-index $\beta=\left(\beta_{1}, \ldots, \beta_{n}\right), \beta \succeq 0$ means that

Received January 25, 2012.

2010 Mathematics Subject Classification. Primary 47B35, 47B38.

Key words and phrases. Toeplitz operator, reducing subspace, Bergman space.

This work was supported by NNSF of China (11201438, 11271059, 10971020), Shanclong Province Young Scientist Research Award Fund (BS2012SF031) and the Fundamental Research Funds for the Central Universities (201213011). 
$\beta_{i} \geq 0$ for any $i \geq 0$. Denote by $z^{\beta}=z_{1}^{\beta_{1}} z_{2}^{\beta_{2}} \cdots z_{n}^{\beta_{n}}$ and

$$
e_{\beta}=\frac{z^{\beta}}{\gamma_{\beta_{1}} \cdots \gamma_{\beta_{n}}},
$$

then $\left\{e_{\beta}\right\}_{\beta}$ is an orthogonal basis in $A_{\alpha}^{2}\left(D^{n}\right)$.

Let $P$ be the Bergman orthogonal projection from $L^{2}\left(D^{n}\right)$ onto $A_{\alpha}^{2}\left(D^{n}\right)$. For a bounded measurable function $f \in L^{\infty}\left(D^{n}\right)$, the Toeplitz operator with symbol $f$ is defined by $T_{f} h=P(f h)$ for every $h \in A_{\alpha}^{2}\left(D^{n}\right)$.

Recall that in a Hilbert space $\mathcal{H}$, a (closed) subspace $\mathcal{M}$ is called a reducing subspace of the operator $T$ if $T(\mathcal{M}) \subseteq \mathcal{M}$ and $T^{*}(\mathcal{M}) \subseteq \mathcal{M}$. A nontrivial reducing subspace $\mathcal{M}$ is said to be minimal if the only reducing subspaces contained in $\mathcal{M}$ are $\mathcal{M}$ and $\{0\}$. On the Bergman space $A_{\alpha}^{2}(D)$, the reducing subspaces of the Toeplitz operators with finite Blaschke product simples are well studied (see $[1,2,8]$ for example). On $A_{\alpha}^{2}\left(D^{2}\right)$, Y. Lu and X. Zhou [4] characterized the reducing subspaces of Toeplitz operators $T_{z_{1}^{N} z_{2}^{N}}, T_{z_{1}^{N}}$ and $T_{z_{2}^{N}}$.

In this note, we consider the reducing subspaces of the Toeplitz operators $T_{z_{1}^{N} z_{2}^{M}}$ on $A_{\alpha}^{2}\left(D^{2}\right)$ and $T_{z_{i}^{N} z_{j}^{M}}$ on $A_{\alpha}^{2}\left(D^{n}\right)$, where $N, M \geq 1$ are integers and $1 \leq i<j \leq n$. Usually, the Toeplitz operators on the unweighted Bergman space and the weighted Bergman space have similar properties (see $[5,6,7,9]$ for example). However, we obtain that the minimal reducing subspaces of $T_{z_{1}^{N} z_{2}^{M}}$ with $N \neq M$ on $A_{\alpha}^{2}\left(D^{2}\right)(\alpha \neq 0)$ are less then that on $A^{2}\left(D^{2}\right)$ (see Theorem 2.4 and Theorem 3.2).

\section{The results on the Bergman space}

Let $M, N$ be integers with $M, N \geq 1$ and $M \neq N$. In this section, we consider the minimal reducing subspace of $T_{z_{1}^{N} z_{2}^{M}}$ on $A^{2}\left(D^{2}\right)$. Here $\gamma_{k}=$ $\left\|z^{k}\right\|_{0}=\sqrt{\frac{1}{k+1}}$. Let $\rho_{1}(k)=\frac{(k+1) N}{M}-1$ and $\rho_{2}(k)=\frac{(k+1) M}{N}-1$. Let $\mathcal{H}_{n m}=$ $\operatorname{Span}\left\{z_{1}^{n} z_{2}^{m}, z_{1}^{\rho_{1}(m)} z_{2}^{\rho_{2}(n)}\right\}$ and $P_{n m}$ be the orthogonal projection from $A_{\alpha}^{2}\left(D^{2}\right)$ onto $\mathcal{H}_{n m}$.

Lemma 2.1. Let $n, m, h$ be nonnegative integers. Then the following statements hold:

(a) if $\rho_{1}(m)$ is an integer, then $\rho_{1}(m+h M)=\rho_{1}(m)+h N$ is an integer for every $h \geq 0$;

(b) if $\rho_{2}(n)$ is an integer, then $\rho_{2}(n+h N)=\rho_{2}(n)+h M$ is an integer for every $h \geq 0$;

(c) if $\rho_{1}(m)$ and $\rho_{2}(n)$ are positive integers, then $\gamma_{\rho_{1}(m)} \gamma_{\rho_{2}(n)}=\gamma_{m} \gamma_{n}$;

(d) $\rho_{1}\left(\rho_{2}(n)\right)=n$ and $\rho_{2}\left(\rho_{1}(m)\right)=m$.

Proof. Notice that if $\rho_{1}(m)$ and $\rho_{2}(n)$ are positive integers, then $\gamma_{\rho_{1}(m)}=$ $\sqrt{\frac{M}{N}} \gamma_{m}$ and $\gamma_{\rho_{2}(n)}=\sqrt{\frac{N}{M}} \gamma_{n}$. So (c) holds. By the direct calculation, (a), (b) and (d) are obvious. 
Theorem 2.2. Let $n, m$ be integers such that $0 \leq n \leq N-1$ or $0 \leq m \leq M-1$, and both of $\rho_{1}(m)$ and $\rho_{2}(n)$ are integers. Then for $a, b \in \mathbb{C}$,

$$
\mathcal{M}=\operatorname{Span}\left\{a z_{1}^{n+h N} z_{2}^{m+h M}+b z_{1}^{\rho_{1}(m+h M)} z_{2}^{\rho_{2}(n+h N)} ; h=0,1,2, \ldots\right\}
$$

is a minimal reducing subspace of $T_{z_{1}^{N} z_{2}^{M}}$ on the polydisk.

Proof. By Lemma 2.1(a) and (b), it is easy to check that $T_{z_{1}^{N} z_{2}^{M}}(\mathcal{M}) \subseteq \mathcal{M}$.

On the other hand,

$$
\begin{aligned}
T_{z_{1}^{N} z_{2}^{M}}^{*}\left(z_{1}^{k} z_{2}^{l}\right) & =\sum_{\beta \succeq 0}\left\langle T_{z_{1}^{N} z_{2}^{M}}^{*} z_{1}^{k} z_{2}^{l}, e^{\beta}\right\rangle e^{\beta} \\
& =\left\{\begin{array}{ccc}
\frac{\gamma_{k}^{2} \gamma_{1}^{2}}{\gamma_{k-N}^{2} \gamma_{l-M}^{2}} z_{1}^{k-N} z_{2}^{l-M}, & \text { if } & k \geq N, l \geq M, \\
0, & \text { if } & \text { others. }
\end{array}\right.
\end{aligned}
$$

For each $h \geq 1$,

$$
\begin{aligned}
& T_{z_{1}^{N} z_{2}^{M}}^{*}\left(z_{1}^{n+h N} z_{2}^{m+h M}\right) \\
= & \frac{\gamma_{n+h N}^{2} \gamma_{m+h M}^{2}}{\gamma_{n+(h-1) N}^{2} \gamma_{m+(h-1) M}^{2}} z_{1}^{n+(h-1) N} z_{2}^{m+(h-1) M}, \\
& T_{z_{1}^{N} z_{2}^{M}\left(z_{1}^{\rho_{1}(m+h M)} z_{2}^{\rho_{2}(n+h N)}\right)}^{*} \\
= & \frac{\gamma_{\rho_{1}(m+h M)}^{2} \gamma_{\rho_{2}(n+h N)}^{2}}{\gamma_{\rho_{1}(m+h M)-N}^{2} \gamma_{\rho_{2}(n+h N)-M}^{2}} z_{1}^{\rho_{1}(m+h M)-N} z_{2}^{\rho_{2}(n+h N)-M} .
\end{aligned}
$$

Combining this with Lemma 2.1(c), it is easy to check that

$$
\begin{aligned}
& T_{z_{1}^{N} z_{2}^{M}}^{*}\left(a z_{1}^{n+h N} z_{2}^{m+h M}+b z_{1}^{\rho_{1}(m+h M)} z_{2}^{\rho_{2}(n+h N)}\right) \\
= & \mu\left(a z_{1}^{n+h N-N} z_{2}^{m+h M-M}+b z_{1}^{\rho_{1}(m+h M-M)} z_{2}^{\rho_{2}(n+h N-N)}\right) \in \mathcal{M},
\end{aligned}
$$

where $\mu=\frac{\gamma_{n+h N}^{2} \gamma_{m+h M}^{2}}{\gamma_{n+(h-1) N}^{2} \gamma_{m+(h-1) M}^{2}}=\frac{\gamma_{\rho_{1}(m+h M)}^{2} \gamma_{\rho_{2}(n+h N)}^{2}}{\gamma_{\rho_{1}(m+h M)-N}^{2} \gamma_{\rho_{2}(n+h N)-M}^{2}(n)}$.

Since $0 \leq n \leq N-1$ (or $0 \leq m \leq M-1)$, we get $\rho_{2}(n)<M$ (or $\rho_{1}(m)<$ $N$, respectively). Therefore, $T_{z_{1}^{N} z_{2}^{M}}^{*}\left(a z_{1}^{n} z_{2}^{m}+b z_{1}^{\rho_{1}(m)} z_{2}^{\rho_{2}(n)}\right)=0 \in \mathcal{M}$. So $T_{z_{1}^{N} z_{2}^{M}}^{*}(\mathcal{M}) \in \mathcal{M}$, which finishes the proof.

Lemma 2.3. Suppose $\mathcal{M} \neq 0$ is a reducing subspace of $T_{z_{1}^{N} z_{2}^{M}}$ in $A^{2}\left(D^{2}\right)$. Let $f=\sum_{(k, l) \succeq 0} a_{k, l} z_{1}^{k} z_{2}^{l} \in \mathcal{M}$. For each nonnegative integers $n, m$ with $a_{n m} \neq 0$, the following statements hold:

(I) if $\rho_{1}(m), \rho_{2}(n)$ are integers and $a_{\rho_{1}(m) \rho_{2}(n)} \neq 0$, then

$$
a_{n m} z_{1}^{n} z_{2}^{m}+a_{\rho_{1}(m) \rho_{2}(n)} z_{1}^{\rho_{1}(m)} z_{2}^{\rho_{2}(n)} \in \mathcal{M}
$$

(II) if at least one of $\rho_{1}(m), \rho_{2}(n)$ is not an integer, or $a_{\rho_{1}(m) \rho_{2}(n)}=0$, then $z_{1}^{n} z_{2}^{m} \in M$. 
Proof. For every integer $h \geq 0$, denote by $T_{h}=T_{z_{1}^{h N} z_{2}^{h M}}$. Notice that

$$
T_{h}^{*} T_{h}\left(z_{1}^{n} z_{2}^{m}\right)=\frac{\gamma_{h N+n}^{2} \gamma_{h M+m}^{2}}{\gamma_{n}^{2} \gamma_{m}^{2}} z_{1}^{n} z_{2}^{m} \in \mathcal{M}, \forall n, m \geq 0 .
$$

Let $P_{\mathcal{M}}$ be the orthogonal projection from $A_{\alpha}^{2}(D)$ onto $\mathcal{M}$, then for nonnegative integers $n, m, k, l$,

$$
\left\langle P_{\mathcal{M}} T_{h}^{*} T_{h} z_{1}^{n} z_{2}^{m}, z_{1}^{k} z_{2}^{l}\right\rangle=\left\langle T_{h}^{*} T_{h} P_{\mathcal{M}} z_{1}^{n} z_{2}^{m}, z_{1}^{k} z_{2}^{l}\right\rangle=\left\langle P_{\mathcal{M}} z_{1}^{n} z_{2}^{m}, T_{h}^{*} T_{h} z_{1}^{k} z_{2}^{l}\right\rangle .
$$

Thus $\frac{\gamma_{h N+k}^{2} \gamma_{h M+l}^{2}}{\gamma_{k}^{2} \gamma_{l}^{2}}=\frac{\gamma_{h N+n}^{2} \gamma_{h M+m}^{2}}{\gamma_{n}^{2} \gamma_{m}^{2}}$. Equivalently,

$$
\frac{(k+1)(l+1)}{(n+1)(m+1)}=\frac{(k+h N+1)(l+h M+1)}{(n+h N+1)(m+h M+1)}, h \geq 0 .
$$
then

$$
(k+1)(l+1)=(n+1)(m+1) .
$$

It follows that $(k+h N+1)(l+h M+1)=(n+h N+1)(m+h M+1)$. Since $g(\lambda)=(k+\lambda N+1)(l+\lambda M+1)-(n+\lambda N+1)(m+\lambda M+1)$ is an analytic polynormal on $\mathbb{C}, g(\lambda)=0$ for any $\lambda \in \mathbb{C}$. The coefficient of $\lambda$ must be zero. We get

$$
M(n-k)=N(l-m) .
$$

This together with (2.3) implies the claim.

Therefore, $P_{\mathcal{M}}\left(z_{1}^{n} z_{2}^{m}\right) \in \mathcal{H}_{n m}$. Hence,

$$
P_{n m} P_{\mathcal{M}}\left(z_{1}^{n} z_{2}^{m}\right)=P_{\mathcal{M}}\left(z_{1}^{n} z_{2}^{m}\right) .
$$

Since $P_{\mathcal{M}} f=f$ for every $f \in \mathcal{M}$, we arrive to

$$
\left\langle P_{\mathcal{M}} P_{n m} f, z_{1}^{n} z_{2}^{m}\right\rangle=\left\langle f, P_{n m} P_{\mathcal{M}} z_{1}^{n} z_{2}^{m}\right\rangle=\left\langle f, P_{\mathcal{M}} z_{1}^{n} z_{2}^{m}\right\rangle=\left\langle P_{n m} f, z_{1}^{n} z_{2}^{m}\right\rangle .
$$

Notice that $\rho_{2}\left(\rho_{1}(m)\right)=m, \rho_{1}\left(\rho_{2}(n)\right)=n$ and $\mathcal{H}_{\rho_{1}(m) \rho_{2}(n)}=\mathcal{H}_{n m}$. Replacing $n, m$ by $\rho_{1}(m)$ and $\rho_{2}(n)$, respectively, it is easy to get that

$$
\left\langle P_{\mathcal{M}} P_{n m} f, z_{1}^{\rho_{1}(m)} z_{2}^{\rho_{2}(n)}\right\rangle=\left\langle P_{n m} f, z_{1}^{\rho_{1}(m)} z_{2}^{\rho_{2}(n)}\right\rangle .
$$

Moreover, $\left\langle P_{\mathcal{M}} P_{n m} f, z_{1}^{k} z_{2}^{l}\right\rangle=\left\langle P_{n m} f, z_{1}^{k} z_{2}^{l}\right\rangle=0$ for any $(k, l) \neq\left(\rho_{1}(m), \rho_{2}(n)\right)$ and $(k, l) \neq(n, m)$. Hence $P_{n m} f=P_{\mathcal{M}} P_{n m}(f) \in \mathcal{M}$. So we get the result.

Theorem 2.4. Suppose $\mathcal{M} \neq\{0\}$ is a reducing subspace of $T_{z_{1}^{N} z_{2}^{M}}$ in the Bergman space $A^{2}\left(D^{2}\right)$. Then there exist $a, b \in \mathbb{C}$ and nonnegative integers $m, n$ with $0 \leq n \leq N-1$ or $0 \leq m \leq M-1$, such that $\mathcal{M}$ contains a reducing subspace as follows

$$
\mathcal{M}_{n, m, a, b}=\operatorname{Span}\left\{a z_{1}^{h N+n} z_{2}^{h M+m}+b z_{1}^{\rho_{1}(m+h N)} z_{2}^{\rho_{2}(n+h M)}: h=0,1,2, \ldots\right\},
$$

where $\rho_{1}(m+h N)=\frac{(m+h N+1) M}{N}-1$ and $\rho_{2}(n+h M)=\frac{(n+h M+1) N}{M}-1$. In particular, if $\rho_{1}(m)\left(\right.$ or $\left.\rho_{2}(n)\right)$ is not a positive integer, then $b=0$. Moreover, $\mathcal{M}$ is minimal if and only if $\mathcal{M}=\mathcal{M}_{n, m, a, b}$. 
Proof. (I) If $\mathcal{M} \neq 0$, there exist nonzero function $f \in \mathcal{M}$ and $k, l$, such that $P_{k l} f \neq 0$. Lemma 2.3 implies that

$$
g_{k l}=P_{k l} f=a z_{1}^{k} z_{2}^{l}+b z_{1}^{\rho_{1}(l)} z_{2}^{\rho_{2}(k)} \in \mathcal{M} .
$$

Observe that there is a positive integer $h_{0}$ such that $a z_{1}^{n} z_{2}^{m}+b z_{1}^{\rho_{1}(m)} z_{2}^{\rho_{2}(n)}=$ $\left(T_{z_{1}^{N} z_{2}^{M}}^{*}\right)^{h_{0}}\left(g_{k l}\right) \neq 0,\left(T_{z_{1}^{N} z_{2}^{M}}^{*}\right)^{h_{0}+1}\left(g_{k l}\right)=0$, where $n=k-h_{0} N, m=l-h_{0} M$. Clearly, $0 \leq n \leq N-1$ or $0 \leq m \leq M-1$. So Theorem 2.2 shows that $a z_{1}^{n} z_{2}^{m}+b z_{1}^{\bar{\rho}_{1}(m)} z_{2}^{\rho_{2}(n)} \in \mathcal{M}_{n, m, a, b} \subseteq \mathcal{M}$.

(II) Suppose $\mathcal{M}$ is minimal. As in (I), there is a nonzero function $a z_{1}^{n} z_{2}^{m}+$ $b z_{1}^{\rho_{1}(m)} z_{2}^{\rho_{2}(n)} \in \mathcal{M}$. Then the following statements hold:

(a) if $z_{1}^{n} z_{2}^{m} \in \mathcal{M}$, then $\mathcal{M}=\operatorname{Span}\left\{z_{1}^{n+h N} z_{2}^{m+h M}, h \geq 0\right\}$;

(b) if $\rho_{1}(m), \rho_{2}(n)$ are integers, and $z_{1}^{\rho_{1}(m)} z_{2}^{\rho_{2}(n)} \in \mathcal{M}$, then

$$
\mathcal{M}=\operatorname{Span}\left\{z_{1}^{\rho_{1}(m)+h N} z_{2}^{\rho_{2}(n)+h M}, h \geq 0\right\} ;
$$

(c) if none of $z_{1}^{n} z_{2}^{m}$ and $z_{1}^{\rho_{1}(m)} z_{2}^{\rho_{2}(n)}$ is in $\mathcal{M}$, then $\mathcal{M}=\mathcal{M}_{n, m, a, b}$ with $a b \neq 0$.

So we finish the proof.

Remark 2.5. Note that

$$
z_{1}^{\rho_{1}(m+h N)} z_{2}^{\rho_{2}(n+h M)}=z_{1}^{m+\frac{(M-N)(m+1)}{N}} z_{2}^{n+\frac{(N-M)(n+1)}{M}} z_{1}^{h M} z_{2}^{h N} .
$$

If $N=M$, then $\rho_{1}(n)=m$ and $\rho_{2}(m)=n$. Y. Lu and X. Zhou [4] showed that $\operatorname{Span}\left\{\left(z_{1}^{k} z_{2}^{m}+z_{1}^{m} z_{2}^{k}\right)\left(z_{1} z_{2}\right)^{h N}: h=0,1,2, \ldots\right\}$ and $\operatorname{Span}\left\{z_{1}^{k} z_{2}^{m}\left(z_{1} z_{2}\right)^{h N}: h=\right.$ $0,1,2, \ldots\}$ are the only minimal reducing subspaces of $T_{z_{1}^{N}} z_{2}^{N}$. Let $a b \neq 0$ with $a \neq b$. Then $\mathcal{M}_{n, m, a, b}$ is a reducing subspace of $T_{z_{1}^{N} z_{2}^{M}}$ when $N \neq M$, but is not a reducing subspace of $T_{z_{1}^{N} z_{2}^{N}}$.

\section{The results on the weighted Bergman space}

Let $-1<\alpha<+\infty$ with $\alpha \neq 0$. In this section, we consider the reducing subspace of $T_{z_{1}^{N} z_{2}^{M}}$ on the weighted Bergman Space $A_{\alpha}^{2}(D)$. Here $\gamma_{n}=\left\|z^{n}\right\|_{\alpha}=$ $\sqrt{\frac{n ! \Gamma(2+\alpha)}{\Gamma(2+\alpha+n)}}$. We begin with a useful lemma.

Lemma 3.1. Let $M, N, n, m, k, l$ be nonnegative integers with $l>m, n>k$ and $M, N \geq 1$. If

$$
\gamma_{h N+k}^{2} \gamma_{h M+l}^{2}=\gamma_{h N+n}^{2} \gamma_{h M+m}^{2}, h \geq 0,
$$

then $N=M, l=n$ and $m=k$.

Proof. First, note that the equality (3.1) holds if and only if for any $\lambda \in \mathbb{C}$ the following equality holds:

$$
\prod_{j=1}^{n-k}(\lambda N+j+k) \prod_{j=1}^{l-m}(\lambda M+2+\alpha+l-j)
$$




$$
=\prod_{j=1}^{n-k}(\lambda N+2+\alpha+n-j) \prod_{j=1}^{l-m}(\lambda M+j+m) .
$$

By computing the coefficient of $\lambda^{n-k+l-m-1}$ in the equality (3.2), we obtain $M \sum_{j=1}^{n-k}(j+k)+N \sum_{j=1}^{l-m}(2+\alpha+l-j)=M \sum_{j=1}^{n-k}(2+\alpha+n-j)+N \sum_{j=1}^{l-m}(j+$ $m)$. It follows that $M(n-k)=N(l-m)$.

Second, we prove that if $\alpha$ is not an integer, then the following statements hold:

$$
(m+1) N=(k+1) M \text { and }(l+1+\alpha) N=(n+1+\alpha) M .
$$

(a) Let $\lambda_{1}=-\frac{k+1}{N}$. Then $\lambda_{1} N+k+1=0$ and $\lambda_{1} N+2+\alpha+n-j \neq 0$ for any $1 \leq j \leq n-k$, because $\lambda_{1} N+2+\alpha+n-j$ is not an integer. Therefore, the equality (3.2) implies that $\prod_{j=1}^{l-m}\left(\lambda_{1} M+j+m\right)=0$. That is, there exists $1 \leq h_{1} \leq l-m$ such that $\lambda_{1} M+m+h_{1}=0$. So, $h_{1}=\frac{k+1}{N} M-m \geq 1$. It follows that $(m+1) N \leq(k+1) M$.

(b) Let $\lambda_{2}=-\frac{m+1}{M}$. Then $\lambda_{2} M+m+1=0$. Similarly, we can get an integer $h_{2}$ such that $1 \leq h_{2} \leq l-m$ and $\lambda_{2} N+k+h_{2}=0$, which implies that $h_{2}=\frac{m+1}{M} N-k \geq 1$. Thus $(m+1) N \geq(k+1) M$.

Comparing (a) with (b), we arrive at $(m+1) N=(k+1) M$.

(c) Let $\mu_{1}=-\frac{n+1+\alpha}{N}$. Then $\mu_{1} N+n+1+\alpha=0, \mu_{1} N+k+j \neq 0$ for any $1 \leq j \leq n-k$. Therefore, $\prod_{j=1}^{l-m}\left(\mu_{1} M+2+\alpha+l-j\right)=0$. That is, there exists $1 \leq h_{3} \leq l-m$ such that $\mu_{1} M+2+\alpha+l-h_{3}=0$. So, $h_{3}=-\frac{n+1+\alpha}{N} M+(2+\alpha+l) \geq 1$, i.e., $(l+1+\alpha) N \geq(n+1+\alpha) M$.

(d) Let $\mu_{2}=-\frac{l+1+\alpha}{M}$. Then $\mu_{2} M+l+1+\alpha=0$. As in (c), there exists $1 \leq h_{4} \leq n-k$ such that $\mu_{2} N+\alpha+2+n-h_{4}=0$. So, $1 \leq h_{4}=$ $-\frac{l+1+\alpha}{M} N+(2+\alpha+n) \leq n-k$ and $(l+1+\alpha) N \leq(n+1+\alpha) M$.

Comparing (c) with (d), we arrive at $(l+1+\alpha) N=(n+1+\alpha) M$.

Third, we prove that if $\alpha$ is an positive integer, then (3.3) holds. In fact, if $1+\alpha \geq 2$ is an integer, then (3.2) can be simplified into

$$
\begin{aligned}
& \prod_{j=1}^{k_{1}}(\lambda N+j+k) \prod_{j=1}^{m_{1}}(\lambda M+2+\alpha+l-j) \\
= & \prod_{j=1}^{k_{1}}(\lambda N+2+\alpha+n-j) \prod_{j=1}^{m_{1}}(\lambda M+j+m), \forall \lambda \in \mathbb{C},
\end{aligned}
$$

where $2 \leq k_{1} \leq n-k, 2 \leq m_{1} \leq l-m, 2+\alpha+n-k_{1}>k_{1}+k$ and $2+\alpha+l-m_{1}>m_{1}+m$. By the same technique as in second part of the proof, we can get the equalities in (3.3).

Finally, combining the equalities (3.3) with $M(n-k)=N(l-m)$, it is easy to get $\alpha N=\alpha M$. Since $\alpha \neq 0$, we have $N=M, l=n, k=m$.

Theorem 3.2. Let $\alpha \neq 0, M, N \geq 1$ with $M \neq N$. Suppose $\mathcal{M} \neq\{0\}$ is a reducing subspace of $T_{z_{1}^{N} z_{2}^{M}}$ in the weighted Bergman space $A_{\alpha}^{2}\left(D^{2}\right)$. Then 
there exist nonnegative integers $n, m$ with $0 \leq n \leq N-1$ or $0 \leq m \leq M-1$ such that

$$
\mathcal{M}_{n m}=\operatorname{Span}\left\{z_{1}^{h N+n} z_{2}^{h M+m}: h=0,1,2, \ldots\right\} \subseteq \mathcal{M} .
$$

In particular, $\mathcal{M}$ is minimal if and only if there exist $n, m$ as in assumption such that $\mathcal{M}=\mathcal{M}_{n m}$.

Proof. Suppose $\mathcal{M} \neq\{0\}$ is a reducing subspace. As in the proof of Lemma 2.3 , there exist integers $n, m$ such that $P_{\mathcal{M}}\left(z_{1}^{n} z_{2}^{m}\right) \neq 0$ and

$$
\frac{\gamma_{h N+k}^{2} \gamma_{h M+l}^{2}}{\gamma_{k}^{2} \gamma_{l}^{2}}=\frac{\gamma_{h N+n}^{2} \gamma_{h M+m}^{2}}{\gamma_{n}^{2} \gamma_{m}^{2}}, \forall h \geq 0,
$$

whenever $\left\langle P_{\mathcal{M}}\left(z_{1}^{n} z_{2}^{m}\right), z_{1}^{k} z_{2}^{l}\right\rangle \neq 0$. Considering that $\left\{\gamma_{j}\right\}_{j=0}^{+\infty}$ is strictly decreasing and $\frac{\gamma_{h N+k}^{2} \gamma_{h M+l}^{2}}{\gamma_{h N+n}^{2} \gamma_{h M+m}^{2}} \rightarrow 1$ as $h \rightarrow+\infty$ [3], we obtain that $\gamma_{k}^{2} \gamma_{l}^{2}=\gamma_{n}^{2} \gamma_{m}^{2}$ and $\gamma_{h N+k}^{2} \gamma_{h M+l}^{2}=\gamma_{h N+n}^{2} \gamma_{h M+m}^{2}, h \geq 0$. This means that one of the following statements holds:

(1) $l=m, n=k$;

(2) $l>m$ and $n>k$;

(3) $l<m$ and $n<k$.

Since $N \neq M$, Lemma 3.1 implies that (2) does not hold. By the same technique, (3) does not hold. So, (1) holds, that is, there exists $c_{n m} \in \mathbb{C}$ such that $P_{\mathcal{M}}\left(z_{1}^{n} z_{2}^{m}\right)=c_{n m} z_{1}^{n} z_{2}^{m}$. For $f=\sum_{(k, l) \succeq 0} a_{k l} z_{1}^{k} z_{2}^{l} \in \mathcal{M}$, we claim that if $a_{n m} \neq 0$, then $c_{n m} \neq 0$. In fact,

$$
\begin{aligned}
Q_{n m} f & =Q_{n m} P_{\mathcal{M}}(f)=Q_{n m}\left(\sum_{(k, l) \succeq 0} P_{\mathcal{M}}\left(a_{k l} z_{1}^{k} z_{2}^{l}\right)\right) \\
& =c_{n m} a_{n m} z_{1}^{n} z_{2}^{m}=c_{n m} Q_{n m} f,
\end{aligned}
$$

where $Q_{n m}$ is the orthogonal projection from $A_{\alpha}^{2}\left(D^{2}\right)$ onto $\operatorname{Span}\left\{z_{1}^{n} z_{2}^{m}\right\}$. Therefore, $c_{n m}=1 \neq 0$.

Hence $z_{1}^{n} z_{2}^{m} \in \mathcal{M}$. Choose an integer $h_{0}$ such that $0 \leq n-h_{0} N \leq N-1$, $m-h_{0} M \geq 0$ or $0 \leq m-h_{0} M \leq M-1, n-h_{0} N \geq 0$. As in the proof of Theorem 2.4, Span $\left\{z_{1}^{n+\left(h-h_{0}\right) N} z_{2}^{m \overline{+}\left(h-h_{0}\right) M}: h=0,1,2, \ldots\right\} \subseteq \mathcal{M}$ is a minimal reducing subspace of $T_{z_{1}^{N} z_{2}^{M}}$. The proof is complete.

Remark 3.3. By the proof of above theorem, we know that on the weighted Bergman space, either $\operatorname{Span}\left\{z_{1}^{n} z_{2}^{m}\right\} \subseteq \mathcal{M}$ or $\operatorname{Span}\left\{z_{1}^{n} z_{2}^{m}\right\} \subseteq \mathcal{M}^{\perp}$ holds.

Theorem 3.4. Let $N, M \geq 1$ and $N \neq M$. Every nonzero reducing subspace $\mathcal{M}$ of $T_{z_{1}^{N} z_{2}^{M}}$ in $A_{\alpha}^{2}\left(D^{2}\right)$ for every $\alpha>-1$ is a direct (orthogonal) sum of some minimal reducing subspaces.

Proof. We prove the theorem in two cases.

Case one: $\alpha \neq 0$. Let us denote

$$
\mathcal{M}_{n m}=\operatorname{Span}\left\{z_{1}^{h N+n} z_{2}^{h M+m}: h=0,1,2, \ldots\right\},
$$


where $0 \leq n \leq N-1$ or $0 \leq m \leq M-1$. By Lemma 3.1, we have $\mathcal{M}_{n m} \subseteq \mathcal{M}$ if and only if there exist some $f \in \mathcal{M}$ with $\left\langle f, z_{1}^{n} z_{2}^{m}\right\rangle \neq 0$. Let $E_{1}=\{(n, m) \succeq$ $0 ; n \leq N-1$ or $m \leq M-1,\left\langle f, z_{1}^{n} z_{2}^{m}\right\rangle \neq 0$ for some $\left.f \in \mathcal{M}\right\}$. Then $\mathcal{M}=$ $\bigoplus_{(n, m) \in E_{1}} \mathcal{M}_{n m}$.

Case two: $\alpha=0$. For $n, m \geq 0$, there exist $a, b \in \mathbb{C}$ such that $\mathcal{M}$ contains the minimal reducing subspace of $T_{z_{1}^{N} z_{2}^{M}}$ defined by

$$
\mathcal{M}_{n, m, a, b}=\operatorname{Span}\left\{a z_{1}^{h N+n} z_{2}^{h M+m}+b z_{1}^{\rho_{1}(m+h N)} z_{2}^{\rho_{2}(n+h M)}: h=0,1,2, \ldots\right\} .
$$

In fact,

(1) If $z_{1}^{n} z_{2}^{m} \in \mathcal{M}$, then $\mathcal{M}_{n, m, 1,0}=\mathcal{M}_{n m}$.

(2) If $z_{1}^{\rho_{1}(m)} z_{2}^{\rho_{2}(n)} \in \mathcal{M}$, then $\mathcal{M}_{n, m, 0,1}=\mathcal{M}_{\rho_{1}(m) \rho_{2}(n)}$.

(3) If neither $z_{1}^{n} z_{2}^{m}$ nor $z_{1}^{\rho_{1}(m)} z_{2}^{\rho_{2}(n)}$ are in $\mathcal{M}$, and there exists $f \in \mathcal{M}$ such that $P_{n m} f \neq 0$, then Theorem 2.4 implies that $\mathcal{M}_{n, m, a, b} \subseteq \mathcal{M}$ is a minimal reducing subspace of $T_{z_{1}^{N} z_{2}^{M}}$, where $P_{n m} f=a z_{1}^{n} z_{2}^{m}+$ $b z_{1}^{\rho_{1}(m)} z_{2}^{\rho_{2}(n)}$. It follows that $P_{n m} g=\lambda\left(a z_{1}^{n} z_{2}^{m}+b z_{1}^{\rho_{1}(m)} z_{2}^{\rho_{2}(n)}\right)$ for every $g \in \mathcal{M}$ with $P_{n m} g \neq 0$.

(4) If $P_{n m} f=0$ for any $f \in \mathcal{M}$, then $\mathcal{M}_{n, m, a, b} \subseteq \mathcal{M}$ if and only if $a=0, b=0$, i.e., $\mathcal{M}_{n, m, 0,0}=\{0\}$.

Let $\mathcal{M}^{\prime}=\mathcal{M} \ominus \mathcal{M}_{n, m, a, b}$. Then $\mathcal{M}^{\prime}$ is a reducing subspace. Continuing this process, since $A^{2}\left(D^{2}\right)=\bigoplus_{(n, m) \succeq 0} z_{1}^{n} z_{2}^{m}$, it is not different to prove that $\mathcal{M}$ is the direct (orthogonal) sum of some minimal reducing subspaces as $\mathcal{M}_{n, m, a, b}$.

In [8], Kehe Zhu shows that a reducing subspace of $T_{z^{N}}$ on $A^{2}(D)$ is the direct (orthogonal) sum of at most $N$ minimal reducing subspaces. However, the reducing subspace of $T_{z_{1}^{N} z_{2}^{M}}$ on $A^{2}\left(D^{2}\right)$ may be the direct (orthogonal) sum of infinity numbers of minimal reducing subspaces. For example, $\mathcal{M}=$ $\operatorname{Span}\left\{z_{1}^{1+2 h} f\left(z_{2}\right) ; f \in A_{\alpha}^{2}(D), h=0,1,2, \ldots\right\}$ is a reducing subspace of $T_{z_{1}^{2} z_{2}^{3}}$ and $\mathcal{M}=\bigoplus_{n=0}^{+\infty} \mathcal{M}_{n}$, where $\mathcal{M}_{n}=\operatorname{Span}\left\{z_{1}^{1+2 h} z_{2}^{n+3 h} ; h=0,1,2, \ldots\right\}$.

\section{The results on the polydisk $A_{\alpha}^{2}\left(D^{n}\right)$}

In this section, we consider the reducing subspace of $T_{z_{i}^{N} z_{j}^{M}}$ in the weighted Bergman space $A_{\alpha}^{2}\left(D^{n}\right)$ with $N \neq M$.

Theorem 4.1. Suppose $\mathcal{M} \neq\{0\}$ is a reducing subspace of $T_{z_{i}^{N} z_{j}^{M}}(N, M \geq$ $1, N \neq M, i \neq j)$ in the weighted Bergman space $A_{\alpha}^{2}\left(D^{n}\right)$. Then the following statements hold:

(a) if $\alpha=0$, then there exist functions $g_{1}, g_{2} \in A_{\alpha}^{2}\left(D^{n-2}\right)$ and integers $l, m$ with $0 \leq l \leq N-1$ or $0 \leq m \leq M-1$, such that $\mathcal{M}$ contains the reducing subspace

$$
\mathcal{M}^{\prime}=\operatorname{Span}\left\{\left(g_{1}\left(z^{\prime}\right) z_{1}^{h N+l} z_{2}^{h M+m}+g_{2}\left(z^{\prime}\right) z_{1}^{\rho_{1}(l+h N)} z_{2}^{\rho_{2}(m+h M)}\right) ; h \geq 0\right\} ;
$$


(b) if $\alpha \neq 0$, then there exist a function $g \in A_{\alpha}^{2}\left(D^{n-2}\right)$ and integers $l, m$ with $0 \leq l \leq N-1$ or $0 \leq m \leq M-1$ such that $\mathcal{M}$ contains the reducing subspace

$$
\mathcal{M}_{l m g}=\operatorname{Span}\left\{z_{i}^{h N+l} z_{j}^{h M+m} g\left(z^{\prime}\right): h=0,1,2, \ldots\right\}
$$

where $z^{\prime}=\left(z_{1}, \ldots, z_{i-1}, z_{i+1}, \ldots, z_{j-1}, z_{j+1}, \ldots, z_{n}\right)$.

Moreover, $\mathcal{M}^{\prime}$ is the only minimal reducing subspace of $T_{z_{i}^{N} z_{j}^{M}}$ on $A^{2}\left(D^{n}\right)$ and $\mathcal{M}_{\text {lmg }}$ is the only minimal reducing subspace of $T_{z_{i}^{N} z_{j}^{M}}$ on $A_{\alpha}^{2}\left(D^{n}\right)$ with $\alpha \neq 0$.

Proof. Without loss of generality, let $i=1$ and $j=2$. Denote by $P_{\mathcal{M}}$ the orthogonal projection from $A_{\alpha}^{2}\left(D^{n}\right)$ onto $\mathcal{M}$. Let $z^{K}=z_{1}^{k_{1}} z_{2}^{k_{2}} \cdots z_{n}^{k_{n}}$ with $P_{\mathcal{M}}\left(z^{K}\right) \neq 0$. Let $T_{h}=T_{z_{1}^{h N} z_{2}^{h M}}$. Then $\left\langle T_{h}^{*} T_{h} P_{\mathcal{M}} z^{K}, z^{L}\right\rangle=\left\langle P_{\mathcal{M}} T_{h}^{*} T_{h} z^{K}, z^{L}\right\rangle$ for any $z^{L}=z_{1}^{l_{1}} z_{2}^{l_{2}} \cdots z_{n}^{l_{n}}$. Observe that

$$
\left\langle P_{\mathcal{M}} z^{K}, T_{h}^{*} T_{h} z^{L}\right\rangle=\frac{\gamma_{h N+l_{1}}^{2} \gamma_{h M+l_{2}}^{2}}{\gamma_{l_{1}}^{2} \gamma_{l_{2}}^{2}}\left\langle P_{\mathcal{M}} z^{K}, z^{L}\right\rangle
$$

and

Therefore,

$$
\left\langle T_{h}^{*} T_{h} z^{K}, P_{\mathcal{M}} z^{L}\right\rangle=\frac{\gamma_{h N+k_{1}}^{2} \gamma_{h M+k_{2}}^{2}}{\gamma_{k_{1}}^{2} \gamma_{k_{2}}^{2}}\left\langle z^{K}, P_{\mathcal{M}} z^{L}\right\rangle
$$

$$
\frac{\gamma_{h N+k_{1}}^{2} \gamma_{h M+k_{2}}^{2}}{\gamma_{k_{1}}^{2} \gamma_{k_{2}}^{2}}=\frac{\gamma_{h N+l_{1}}^{2} \gamma_{h M+l_{2}}^{2}}{\gamma_{l_{1}}^{2} \gamma_{l_{2}}^{2}}, \forall h \geq 0,
$$

whenever $\left\langle P_{\mathcal{M}} z^{K}, z^{L}\right\rangle \neq 0$.

If $\alpha=0$, then as in Lemma 2.3 we have $\left(l_{1}, l_{2}\right)=\left(k_{1}, k_{2}\right)$ or $\left(l_{1}, l_{2}\right)=$ $\left(\rho_{1}\left(k_{2}\right), \rho_{2}\left(k_{1}\right)\right)$ where $\rho_{1}\left(k_{2}\right), \rho_{2}\left(k_{1}\right)$ are integers. Thus $P_{\mathcal{M}} z_{1}^{\rho_{1}\left(k_{2}\right)} z_{2}^{\rho_{2}\left(k_{1}\right)} z^{\prime K^{\prime}}$ and $P_{\mathcal{M}} z^{K}$ are in $z_{1}^{k_{1}} z_{2}^{k_{2}} A^{2}\left(D^{n-2}\right)+z_{1}^{\rho_{1}\left(k_{2}\right)} z_{2}^{\rho_{2}\left(k_{1}\right)} A^{2}\left(D^{n-2}\right)$, where $z^{\prime}=\left(z_{3}\right.$, $\left.\ldots, z_{n}\right)$ and $K^{\prime}=\left(k_{3}, \ldots, k_{n}\right)$. Let $P_{k_{1} k_{2}}$ be the orthogonal projection from $A^{2}\left(D^{n}\right)$ onto

$$
\operatorname{Span}\left\{z_{1}^{k_{1}} z_{2}^{k_{2}} A^{2}\left(D^{n-2}\right)+z_{1}^{\rho_{1}\left(k_{2}\right)} z_{2}^{\rho_{2}\left(k_{1}\right)} A^{2}\left(D^{n-2}\right) ; h=0,1,2, \ldots\right\} .
$$

Then $P_{k_{1} k_{2}} P_{\mathcal{M}} z^{K}=P_{\mathcal{M}} P_{k_{1} k_{2}} z^{K}$. For each $f \in \mathcal{M}$ with $f \neq 0$, there are integers $l, m \geq 0$ such that $P_{l m} f \neq 0$. By the similar technique, we can proof that $\left\langle P_{\mathcal{M}} P_{m l} f, z^{K}\right\rangle=\left\langle P_{m l} f, z^{K}\right\rangle$ for any $K \succeq 0$, i.e., $P_{\mathcal{M}} P_{m l} f=P_{m l} f$. So, there exist $f_{1}\left(z^{\prime}\right)$ and $g_{2}\left(z^{\prime}\right) \in A^{2}\left(D^{n-2}\right)$ such that $P_{m l} f=g_{1}\left(z^{\prime}\right) z_{1}^{m} z_{2}^{l}+$ $g_{2}\left(z^{\prime}\right) z_{1}^{\rho_{1}(l)} z_{2}^{\rho_{2}(m)} \in \mathcal{M}$, which implies that (a) holds.

If $\alpha \neq 0$, then we arrive at $P_{\mathcal{M}} z^{K} \in z_{1}^{k_{1}} z_{2}^{k_{2}} A_{\alpha}^{2}\left(D^{n-2}\right)$. Denote by $P_{k_{1} k_{2}}^{\prime}$ the orthogonal projection from $A_{\alpha}^{2}\left(D^{n}\right)$ onto

$$
\operatorname{Span}\left\{z_{1}^{k_{1}} z_{2}^{k_{2}} A^{2}\left(D^{n-2}\right) ; h=0,1,2, \ldots\right\} .
$$

Then $P_{k_{1} k_{2}}^{\prime}(f)=P_{k_{1} k_{2}}^{\prime} P_{\mathcal{M}}(f)=P_{\mathcal{M}} P_{k_{1} k_{2}}^{\prime}(f) \in \mathcal{M}$ for each $f \in \mathcal{M}$. Hence (b) holds. The rest of the proof is obvious. 


\section{References}

[1] K. Guo and H. Huang, On Multiplication operators on the Bergman space: similarity, unitary equivalence and reducing subspaces, J. Operator Theory 65 (2011), no. 2, 355378.

[2] K. Guo, S. Sun, D. Zheng, and C. Zhong, Multiplication operators on the Bergman space via the Hardy space of the bidisk, J. Reine Angew. Math. 628 (2009), 129-168.

[3] Y. Lu and Y. Shi, Hyponormal Toeplitz operators on the weighted Bergman space, Integral Equations Operator Theory 65 (2009), no. 1, 115-129.

[4] Y. Lu and X. Zhou, Invariant subspaces and reducing subspaces of weighted Bergman space over bidisk, J. Math. Soc. Japan 62 (2010), no. 3, 745-765.

[5] S. Shimorin, On Beurling-type theorems in weighted $l^{2}$ and Bergman spaces, Proc. Amer. Math. Soc. 131 (2003), no. 6, 1777-1787.

[6] L. Trieu, On Toeplitz operators on Bergman spaces of the unit polydisk, Proc. Amer. Math. Soc. 138 (2010), no. 1, 275-285.

[7] X. Zhou, Y. Shi, and Y. Lu, Invariant subspaces and reducing subspaces of weighted Bergman space over polydisc, Sci. Sin. Math. 41 (2011), no. 5, 427-438.

[8] K. Zhu, Reducing subspaces for a class of multiplication operators, J. London Math. Soc. 62 (2000), no. 2, 553-568.

[9] _ Operator Theory in Function Spaces, 2nd ed. Providence, R.I.: American Mathematical Society, 2007.

YANYUe ShI

College of Mathematical Science

OCEAN University of China

QIngdaO, 266100, P. R. CHINA

E-mail address: shiyanyue@gmail.com

YUFENG LU

School of Mathematical Sciences

Dalian University of TeChNOLOGY

Dalian, 116024, P. R. China

E-mail address: lyfdlut@dlut.edu.cn 